\title{
(3) Pathogenetic Significance of Antinuclear Factor in Systemic Lupus Erythematosus
}

\author{
Tadashi OfuJI \\ The Third Department of Internal Medicine, \\ Okayama University Medical School
}

Immunoserological studies on systemic lupus erythematosus (SLE) and immunopathological observations on lupus nephritis have been performed to clarify the pathogenetic significance of antinuclear factor (ANF) in SLE.

Patients :

Seventy cases of SLE admitted to this clinic between April, 1967 and March, 1972.

Methods :

ANF : Indirect fluorescent antibody technique, using peripheral leukocytes as nuclear materials.

DNA antibody: Carr-Kunkel's Actinomycin D-DNA method.

Anticomplementary activity (ACA): Consumption rate of complement measured by EA-cells added with rat complement and inactivated serum to be tested.

Immunopathological examination: Staining of biopsy and autopsy materials with fluorescent anti-human $\operatorname{IgG}, \mathrm{A}, \mathrm{M}$ and $\beta 1 \mathrm{c} / \beta 1 \mathrm{~A}$ sera.

Results :

1. Serological abnormal findings

Out of 2,691 different specimens, 1,084 specimens gave positive reaction to ANF. Seventy-two per cent of them were SLE and 28 per cent non-SLE. All of 82 different specimens of SLE were positive for ANF.

Staining patterns observed by the indirect fluorescent antibody technique for ANF were classified into shaggy pattern ( $s-p)$, diffuse pattern (d-p) and speckled pattern (sp-p). It was found that $s-p$ corresponded to DNA antibody, d-p to NP antibody and sp-p to PBFS antibody.

Out of 1,084 different specimens that were positive for ANF, 410 specimens showed s-p, 95 per cent of them being SLE. This meant that s-p was highly specific for SLE.

Among the DNA-antibody detecting methods, Actinomycin D-DNA method is excellent to check out a small amount of DNA which can not be found by precipitation or complement fixation methods. The level of DNA measured by this method 
was markedly high in the acute stage of SLE.

CH50 was markedly low in the acute stage of SLE. ACA was high in the acute stage accompanied with active lupus nephritis, showed a close correlation with DNA antibody level and decreased after DNase treatment.

When complement fixation ability was examined by immune complex produced from added DNA and sera of patients with and without active lupus nephritis, sera from the "active" group showed a strong complement fixation ability. When thus produced complex was intracutaneously injected and Evans blue intracardially injected in rats, blue spots produced were measured to be larger in the "active" group.

From results obtained, it can be postulated that immune complex, mainly composed of DNA antibody, is present in the blood and DNA anti-DNA antibody complex found particularly in active lupus nephritis is stronger in complement fixation ability and in tissue damage than that without active lupus nephritis.

2. Immunopathological studies on lupus nephritis

Forty-six specimens of renal tissue of lupus nephritis were immunopathologically examined chiefly by the fluorescent antibody technique and compared with abnormal findings of serum. A modified criterion of Schur was used for the degree of activity of the nephritis.

Eighty-eight per cent of the "active" group showed more than 20 per cent in DNA antibody level, less than 20 in $\mathrm{CH} 50$ and strong serum abnormality. Only 17 per cent of the "inactive" group and 7 per cent of the "without" group revealed the same serum abnormality.

Staining patterns of lupus nephritis by the fluorescent antibody technique can be classified into 4 groups, i.e. linear pattern (lin-p), mesangial pattern (mes-p), granular pattern (gr-p) and lumpy pattern (lum-p). Cases showing gr-p by the fluorescent antibody technique displayed corresponding deposits by electron microscopy in which $\gamma$-globulin was demonstrated by the electron microscopic enzymelabeled antibody technique.

The majority of gr-p and lum-p were seen in active lupus nephritis and many of lin-p and mes-p in the "without" group.

In mes-p, gr-p and lum-p showing deposits, DNA antibody level was more than 20 per cent, CH50 less than 20 per cent and strong serum abnormality was seen. In lin-p which showed no deposits, DNA antibody level was subject to be less than 20 per cent and CH50 more than 20.

With correlation between the fluorescent antibody staining of lupus nephritis and immunoglobulin, $\operatorname{IgG}$ is the most, followed by $\operatorname{IgM}$, and $\operatorname{Ig} \mathrm{A}$ the least. $\beta 1 \mathrm{c} / \beta 1 \mathrm{~A}$ was found to be 100 per cent in mes-p, gr-p and lum-p, while it was negative in all cases of lin-p.

After renal tissue was treated with acid buffer at $\mathrm{pH} 3.2$, mes-p, gr-p and lum-p showed a decrease in fluorescence. Some of eluates were demonstrated to contain ANF. Hence, it was strongly postulated that a portion, at least, of the deposits 
was ANF-nuclear substance complex.

Conclusion :

From the above-mentioned results, it is postulated that the deposits of lupus nephritis are derived from immune complex in the circulating blood. However, the question whether or not the pathogenesis of SLE can be solved completely with immune complex theory remains to be clarified at the present time. 